\title{
Automatic localization of the optic disc by combining vascular and intensity information
}

\author{
Ana Maria Mendonça ${ }^{\mathrm{a}, \mathrm{b}, *}$, António Sousa ${ }^{\mathrm{a}, \mathrm{c}}$, Luís Mendonça ${ }^{\mathrm{d}}$, Aurélio Campilho ${ }^{\mathrm{a}, \mathrm{b}}$ \\ a INEB - Instituto de Engenharia Biomédica, Universidade do Porto, Porto, Portugal \\ ${ }^{\mathrm{b}}$ FEUP - Faculdade de Engenharia, Universidade do Porto, Porto, Portugal \\ c ISEP - Instituto Superior de Engenharia, Instituto Politécnico do Porto, Porto, Portugal \\ d Serviço de Oftalmologia - Hospital de Braga, Braga, Portugal
}

\section{A R T I C L E I N F O}

Article history:

Received 7 December 2012

Received in revised form 19 April 2013

Accepted 25 April 2013

\section{Keywords:}

Retinal image analysis

Optic disc localization

Retinal vessel segmentation

Entropy

\begin{abstract}
A B S T R A C T
This paper describes a new methodology for automatic location of the optic disc in retinal images, based on the combination of information taken from the blood vessel network with intensity data. The distribution of vessel orientations around an image point is quantified using the new concept of entropy of vascular directions. The robustness of the method for OD localization is improved by constraining the search for maximal values of entropy to image areas with high intensities. The method was able to obtain a valid location for the optic disc in 1357 out of the 1361 images of the four datasets.
\end{abstract}

(c) 2013 Elsevier Ltd. All rights reserved.

\section{Introduction}

The optic disc (OD) is one of the major landmarks that stand out from the observation of a human retina. OD localization is frequently required for the implementation of image analysis-based solutions for the assessment and follow-up of several eye conditions. Detection of the OD is relevant in glaucomatous eyes as this disorder affects the shape and appearance of the disc, but it can also be useful for differentiating from white lesions related to diabetic retinopathy, or from drusen associated with age-related macular degeneration [1]. Knowledge about OD position is also required for automating the determination of diagnostic indexes for hypertensive retinopathy, such as the Arteriolar-to-Venular diameter ratio (AVR) [2].

The OD is the entry point of the nervous and blood systems into the retina and is often the brightest region in a fundus image. These two distinctive features have been the major strategies for automating the localization and detection of the OD reported in the literature. Approaches that rely on features derived from color include the use of maximal intensity, maximal variance, or the evaluation of image variation along specific directions [3-6], template-matching $[7,8]$, Hough transform $[4,10]$ and deformable

\footnotetext{
* Corresponding author at: INEB, Campus da FEUP, Rua Dr. Roberto Frias, s/n, 4200-465 Porto, Portugal. Tel.: +351 225081859.

E-mail addresses: amendon@fe.up.pt (A.M. Mendonça), asousa@eu.ipp.pt (A. Sousa), luismendonc@gmail.com (L. Mendonça), campilho@fe.up.pt (A. Campilho).
}

models [8,9]. Several authors have proposed methods that use the blood vessel network as a starting point for OD detection [11-15]. Solutions combining the simultaneous detection of several retinal structures are described in $[16,17]$.

This paper describes a method for automating the localization of the OD in color eye fundus images, by combining information extracted from the vascular network with intensity data obtained from the red $(R)$ and green $(G)$ channels of the RGB representation. The distribution and variability of vessels around each image point are estimated using the concept of entropy of vascular directions, which associates high values of this measure with the occurrence of a large number of vessels with multiple orientations. This information is then combined with the highest image intensities, with the goal of localizing pixels where both entropy and intensity are maximized. The here in proposed approach is an improved and extended version of the method previously presented by the authors of a conference paper in [18]. The major differences between the two solutions are: the initial calculation of the entropy of vascular directions using a low resolution image; the use of the entropy map for evaluating the quality of the vascular segmentation and deciding on the criteria for estimating the localization of the disc; and the inclusion of a post-processing step for obtaining the final OD position.

The paper is organized as follows. Section 2 describes the methodology that was developed for automating the localization of the disc. The results obtained using the images of four publicly available databases, DRIVE [19], STARE [20], MESSIDOR [21], and INSPIRE-AVR [22], are reported in Section 3. Finally, Section 4 presents some conclusions. 

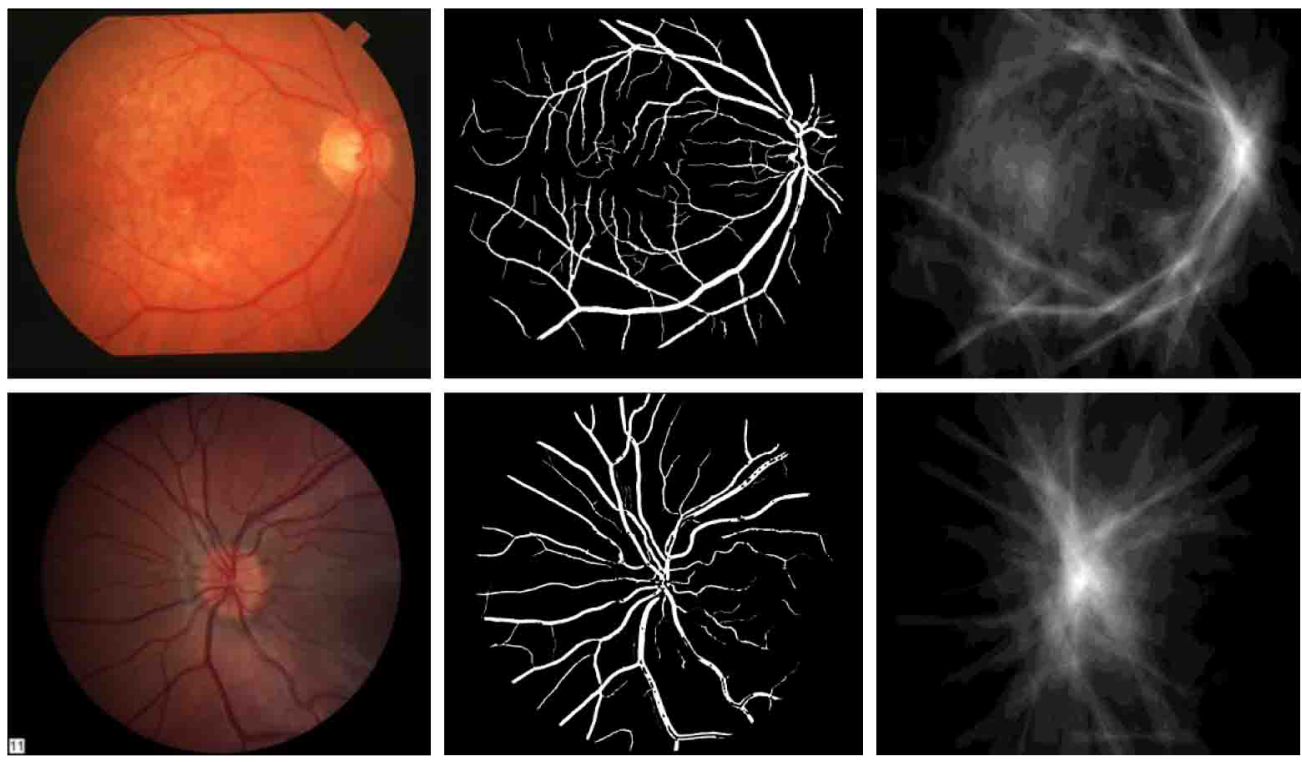

Fig. 1. Original images (left), segmented vascular networks (middle), and entropy maps (right).

\section{Methodology for optic disc localization}

\subsection{Overview of the methodology}

The first phase of the method is the estimation of an initial OD position through the application of the algorithm described in [18] to a low resolution version of the original image. In this method the entropy of vascular directions is used for quantifying both the occurrence and the diversity of vessel orientations around a pixel. After the segmentation of the vascular network, a map with the entropy of vascular directions is calculated for the fundus image. An intensity map representing the Euclidean distance of the red $(\mathrm{R})$ and green $(\mathrm{G})$ components to the origin of the RGB color coordinates is generated from the color image of the retina; this map is afterwards segmented to retain just the pixels with the largest distance values, which are further analyzed to generate a restricted set of high intensity OD candidates. The initial OD location is the candidate where the maximum value of entropy occurs.

The second phase of the algorithm, which is new in the here in proposed solution, is either a validation or a recalculation of the disc position. When values of entropy are low, and in particular the absolute maximum of entropy or the entropy in the estimated OD location, the quality of the vascular segmentation is not reliable and a new estimate for disc position is searched for using mainly intensity criteria. For high values of entropy, the initial OD position is validated if all other local maxima of entropy occurring in different image locations show lower values when compared with the one corresponding to the current estimation for OD position. On the other hand, if several identical local maxima are found, the process for estimating the OD position is repeated once again using the full resolution image. A final post-processing phase is applied to a restricted region around the estimated position of the disc, in order to get the final OD location as the weighted centroid of the region formed by the combination of vessel and intensity segmentation location results. The main processing phases required for locating the $\mathrm{OD}$ are detailed in the following subsections.

\subsection{Entropy of vascular directions}

The OD is a region of the eye fundus where the vessels are more or less distributed in all possible directions. For evaluating the convergence and variability of vessel directions in the neighborhood of each point in a retinal image, a new measure was proposed in [18] based on the definition of entropy proposed by Shannon. The entropy of vascular directions is calculated using (1), where $n$ is the number of directions and $p_{i}$ the probability of occurrence of vessels in direction $i$.

$H=-\sum_{i=1}^{n} p_{i} \log p_{i}$

In order to calculate the entropy map of a retinal image, a direction needs to be associated to every vessel pixel. The segmentation of retinal blood vessels is accomplished using the algorithm proposed in [23], and to obtain the vessel direction information a matchedfiltering approach similar to the one described in [24] is applied. For this purpose a grey-scale version of the original RGB image is sequentially processed with twelve two-dimensional matched filters, each one adapted to a specific direction; the highest filtering output for a vessel point defines the direction of the vessel at that point.

The entropy map is determined based on the normalized histogram of vessel directions in a window centered on each image point. For every vessel pixel inside this window, two directions are considered: an absolute direction, taken from the vessel direction map, and a relative direction which is the direction of the vector that links the vessel pixel to the window center. The histogram of vessel directions only retains information for those vessel points whose absolute and relative directions are coincident.

Fig. 1 shows two original retinal images, their segmented vascular networks and the corresponding entropy maps. As can be observed, entropy values clearly increase near and inside vessels and in particular in the OD region.

To calculate the entropy at each image point, a histogram of vessel directions needs to be determined in a window centered at that point. The size of this window should be large enough to capture the diversity of vessel distribution around the point, and small enough to keep processing time low. In [18] a window size of $351 \times 151$ pixels was set for the images of the DRIVE and STARE databases. In the method herein described, the images are initially reduced to half in each dimension and the window for entropy calculation is decreased accordingly to [ $175 \times 75]$ pixels. In both cases, rectangular windows oriented towards the vertical direction are chosen due to the expected distribution of blood vessel near the OD, which 


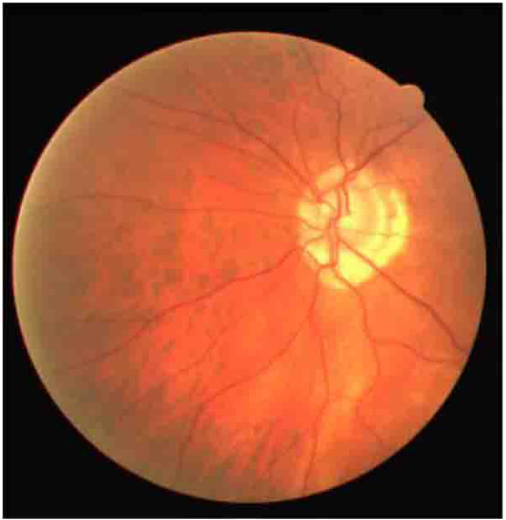

(a)

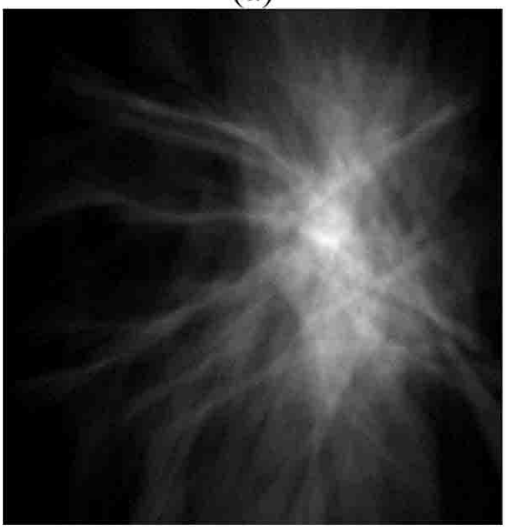

(c)

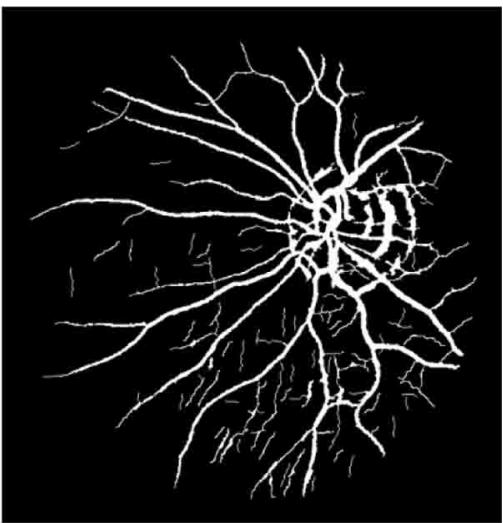

(b)

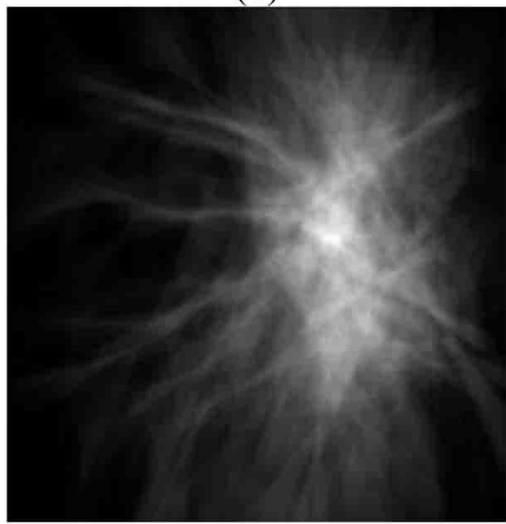

(d)

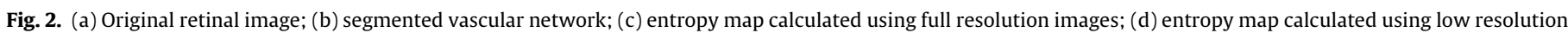
images.

tend to spread mainly in the vertical direction before forming the two main vessel arcades around the macula. As a result, the entropy values on these rectangular windows are more selective than those obtained using square neighborhoods. Moreover, an increased discrimination between the OD area and other retinal regions where relevant concentrations of vessels occur is achieved.

Entropy maps for both the original retinal image and its sizereduced version are presented in Fig. 2. As can be observed, no overall significant differences can be found between the two entropy maps, although a more detailed analysis of local distribution of values can reveal sharper local maxima for the full resolution image. Despite this, in good quality images the low resolution entropy values have no influence on the final calculated OD position.

\subsection{Initial estimation of $O D$ location}

The direct calculation of the absolute maximum of entropy is sufficient for estimating the OD location in most retinal images. However, when the vessels around the disc are not well segmented, mainly due to poor image quality or pathological conditions, the entropy map contains several local maxima and the simple detection of the largest value may not be able to identify the correct OD location. In order to restrict the areas in which the local maximum of entropy is searched for, bright intensity, which is another characteristic feature of a normal OD, is taken into account.

As the OD is usually a yellowish bright region in a normal image of the retina, and does not contain important contents in the blue channel, we derive an image from the red $(R)$ and green $(G)$ components from the original RGB image, using (2). In this image, I, a pixel value is represented as the Euclidean distance, in the RG plane, from the origin of the color coordinate system.

$I(x, y)=\sqrt{R(x, y)^{2}+G(x, y)^{2}}$

Aiming at reducing the influence of the vessels in the subsequent selection of candidate regions, the image is processed with a closing morphological operator using a structuring element adapted to vessel size. This image is afterwards submitted to illumination equalization, as described by Eq. (3), where $\bar{I}_{W}(x, y)$ is the average intensity of pixels inside a window $\mathrm{W}$ centered on the spatial coordinates $(x, y)$.

$I_{\text {eq }}(x, y)=I(x, y)+0.5-\bar{I}_{W}(x, y)$

Eq. (3) performs an intensity rescaling operation aiming at obtaining an image with a specified average value equal to 0.5 , which corresponds to the central value in the [0-1] range used for representing intensities.

To select an initial set of candidate areas only $10 \%$ of the highest intensity points of image $I_{\mathrm{eq}}$ are kept. As the number of regions is still large, an image-dependent threshold is calculated and applied to obtain the final set of candidate areas where in the maximum value of entropy will be searched for, and then used for obtaining the OD position. The threshold value, $T$, is calculated based on the mean, $m_{\max }$, and standard deviation, $s_{\max }$, of intensity maxima in the connected regions of the initial set, as established in (4). These intensity maxima are taken from the green component of the original RGB image after illumination equalization $\left(G_{\mathrm{eq}}\right)$. This image is used instead of $I_{\mathrm{eq}}$ for threshold estimation because of its higher contrast. The definition of two alternative equations for $T$ is justified by the need to adapt to the distinct image intensity characteristics. 


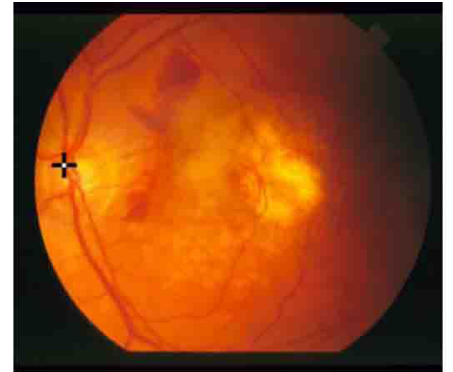

(a)

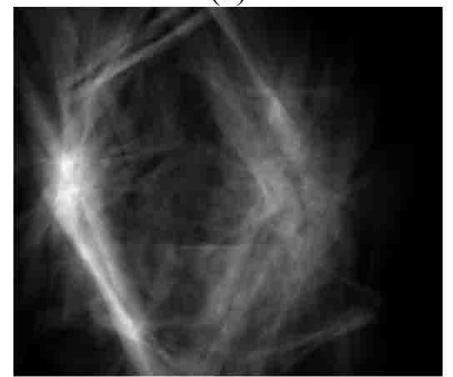

(b)

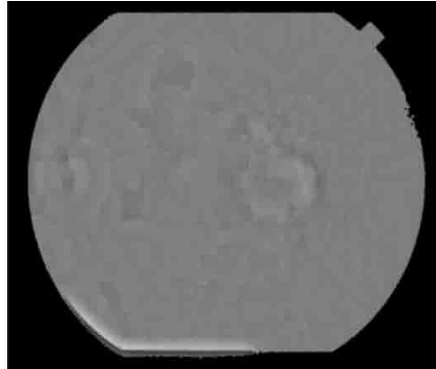

(c)

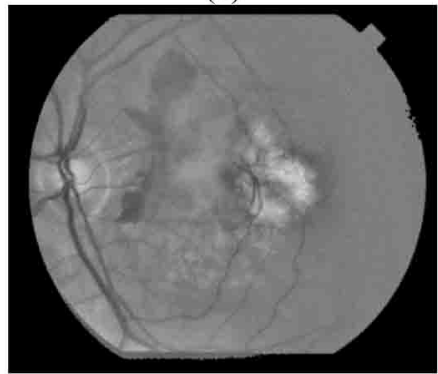

(d)

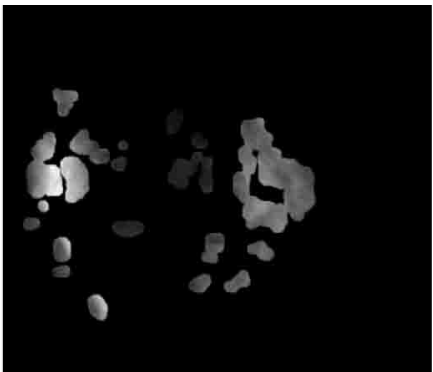

(e)

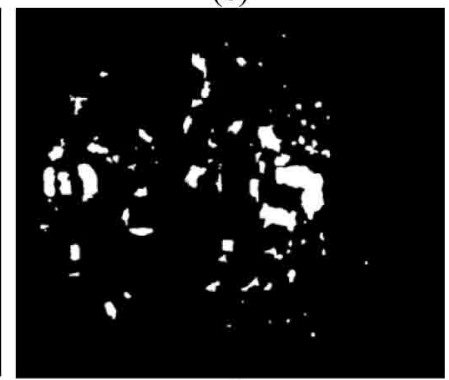

(f)

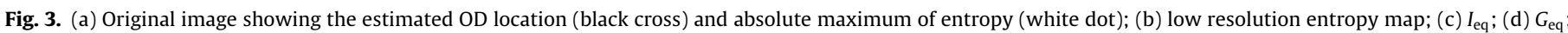
(e) intensity segmented image; (f) final set of segments after dilation with the values of entropy overlapped.

Thresholded areas are dilated using a structuring element adapted to vessel caliber; this widening operation is particularly important near the OD as the vessels have high values of entropy and are not included in the set of candidate areas due to their low intensity values. Finally, the point with the maximum value of entropy is set as the initial estimation of OD location.

$T= \begin{cases}m_{\max } & \text { if } s_{\max }>0.1 m_{\max } \\ m_{\max }+2 s_{\max } & \text { all other cases }\end{cases}$

Final and intermediate results of the processing sequence for attaining an initial OD position are illustrated in Fig. 3. Fig. 3a shows the original image with the estimated OD position (black cross); in this image, the absolute maximum of entropy (white dot) is coincident with the estimated OD location. Fig. 3b-d present the low resolution entropy map, $I_{\mathrm{eq}}$, and $G_{\mathrm{eq}}$, respectively. Finally, the initial and final sets of candidates obtained from the intensity-segmented image are shown in Fig. 3e and f; in this last image, the values of entropy for each selected image pixel are also depicted.

\subsection{Validation of estimated OD location}

The evaluation of the maximum of the entropy proved able to locate the OD in the great majority of retinal images. However, when the vascular structure near the OD is not entirely visible or is affected by some pathology, the search for the absolute maximum of the measure may be insufficient for reaching a suitable final result. Nevertheless, in most situations the search for the maximal value of entropy, combined with the occurrence of high intensities, is adequate for achieving a correct OD location.

In a few situations, especially when the quality of the retinal image is low (mainly due to problems in image acquisition), the segmented vasculature around the disc is either inaccurate or simply missing, and the estimated position of the OD as described in the previous subsection does not hit the optic disc. In other cases, the existence of very intense pathological lesions near the vessels may introduce erroneous segments in the vascular tree and mislead the OD estimation algorithm. Another problem is that the use of low resolution images for entropy calculation can give rise to the existence of several local maxima with similar values in distinct image positions, which, in just a few cases, can prevent the absolute maximum of entropy from being associated with the actual OD location.

In order to cope with these limitations, a validation phase is included. For this purpose, the following properties are evaluated on the low resolution entropy map: (i) the absolute maximum of entropy; (ii) the relation of the entropy value for the estimated OD position to the absolute maximum of entropy; (iii) the presence of other local maxima whose values are similar to the entropy value of the estimated OD position.

The absolute maximum of entropy proved an effective measure for assessing the quality of the vascular segmentation, with low values of this indicator generally associated with unreliable OD estimation. On the other hand, when the absolute maximum value is high, the entropy value for the estimated OD position should not be below an image-dependent threshold value (set as $60 \%$ of the image absolute maximum), to prevent situations where the intensity criterion mostly determines the selected OD location.

When one of these two situations is identified, a recalculation step is initiated, trying to find an alternative solution for OD location. For this purpose, the region of the intensity segmented image with the largest area is found, because although the disk is normally fragmented by the dark blood vessels, at least one of the segments still remains large due to the uniformity of the disc intensity. So a new estimate for OD position is obtained corresponding to the maximal intensity in this region - but this new solution is only kept if this maximum is higher than the intensity of the initial OD position estimation. This last condition is relaxed to a lower limit (95\% of maximum intensity) if the entropy of the largest area is higher than the one in the initially selected position. Finally, when several high intensity local maxima exist in the low resolution entropy map, the initial estimation of OD is not accepted and the algorithm described in the previous section is repeated using the full resolution images.

Examples of images illustrating these situations are presented in Fig. 4, where from left to right we can find the original image, the segmented vasculature, the entropy map and the intensity candidates. For the images of the top row, the OD position was recalculated based on maximal area and intensity criteria, because 

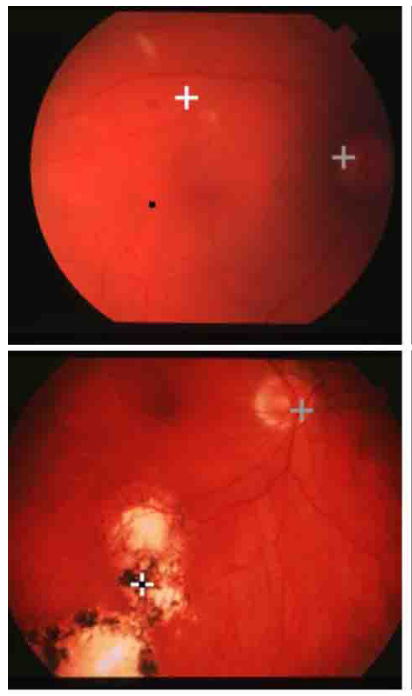

(a)

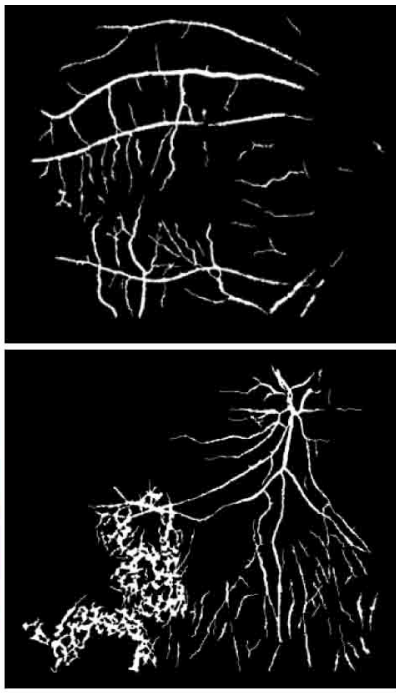

(b)

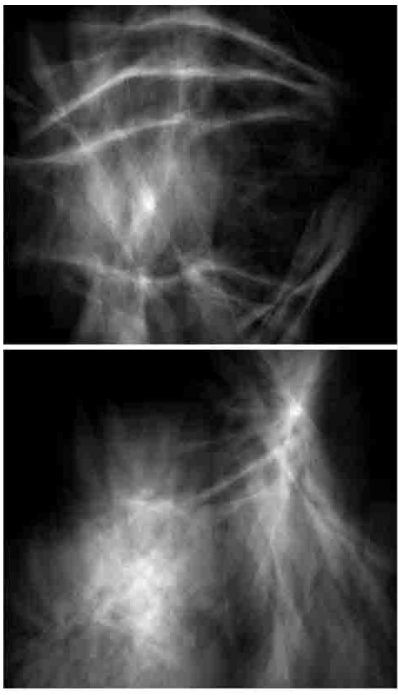

(c)

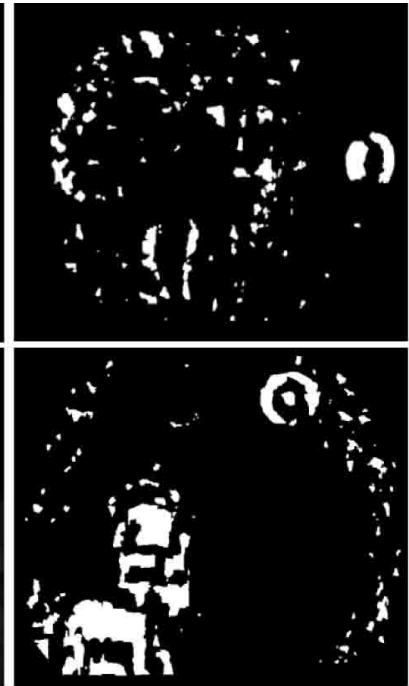

(d)

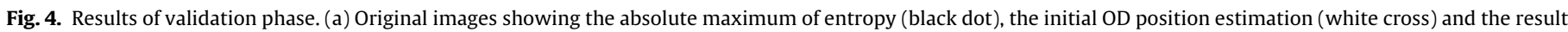

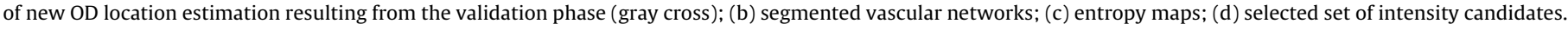

the absolute maximum of entropy obtained in the first phase was below the specified threshold and was considered unreliable; the example depicted in the bottom row presents an image showing several high entropy maxima, where the recalculation of OD position using the full resolution images allowed a correct solution in the validation phase.

\subsection{Refinement of OD location}

The final phase of the proposed methodology aims at refining the disc position previously estimated. This final post-processing phase is applied on a restricted circular region centered on the estimated OD position with diameter equal to 1.5 times the expected disc diameter. The underlying idea is that the region of the disc can be reconstructed if the vessel- and intensity-segmented images are added together. A new value, combining entropy and intensity by their geometric mean, is assigned to each pixel of this new image. The weighted centroid of the circular region centered on the previously estimated OD location is considered the final position of the optic disc center.

The process for obtaining the final OD position is illustrated in Fig. 5. The segmented vasculature (Fig. 5a) and the set of intensity candidates (Fig. 5b) are combined to get the image presented in Fig. 5c. The circular restricted area centered on the estimated OD location, with each pixel weighted by the combination of entropy and intensity values, is shown in Fig. 5d. Finally, Fig. 5e shows the original image with the OD positions before (white cross) and after (black cross) the refinement step.

\section{Results}

The methodology for OD localization was evaluated using the images of four publicly available databases, DRIVE [19], STARE [20], MESSIDOR [21], and INSPIRE-AVR [22]. The DRIVE database consists of 40 color images of the retina $(565 \times 584,24$ bits), where 33 do not show signs of pathology. No information regarding OD position is publicly available for this dataset.

The STARE database contains 81 eye fundus images $(700 \times 605$, 24 bits) that were initially selected by Hoover et al. [11] for evaluating their method for OD localization. This dataset consists of 31 images of normal retinas, and 50 images showing some kind of pathology. The MESSIDOR database consists of 1200 images grouped into three subsets from different ophthalmologic departments. This dataset contains images with distinct sizes $(980 \times 1440$, $1488 \times 2240,1536 \times 2304,24$ bits). All images were acquired using a non-mydriatic retinograph with a $45^{\circ}$ field of view, 800 with and 400 without pupil dilation. The last set of images is formed from the 40 images of the test set of the INSPIRE-AVR database. These high resolution images $(2048 \times 2392,24$ bits $)$ are centered on the disc and were acquired with a $30^{\circ}$ field of view.

The entropy maps used in the first phase of the method were obtained based on vessel direction histograms calculated on a rectangular window of $175 \times 75$ pixels centered in each image pixel. The images of the MESSIDOR and INSPIRE-AVR datasets were initially downsized to dimensions similar to the DRIVE and STARE images (using scale factors of $1 / 1.5$ and $1 / 2.5$ for MESSIDOR, and $1 / 4$ for INSPIRE). Before computation of entropy, all images were once again reduced by half in each dimension. Although computation time is still slightly dependent on the original size of the image, as some of the operations are performed using the full image size (or the size that results from the first scaling step for the Messidor and Inspire-AVR databases), a reduction of $1 / 12$ in the processing time was in general achieved for all databases. For instance, in DRIVE images the time required to process full resolution images (about $90 \mathrm{~s}$ ) was reduced to an average value of $8 \mathrm{~s}$.

The parameter values that required adaptation to each specific dataset in the previous method [18] are now fixed and identical for all databases. The proposed approach only depends on two parameters: one is the scaling factor just mentioned, and the other is the lower limit for the absolute maximum of entropy, which is used to decide whether the entropy map is or is not reliable for OD location, as explained in Section 2.4. While the value of the scaling parameter is automatically derived from the original image size, the limit for entropy is obtained from the average and standard deviation of the entropy maxima for each dataset. Identical limits were used for all databases except MESSIDOR, as entropy values for this set are slightly smaller, because, on average, the number of segmented vessel pixels in the downscaled images is lower.

For evaluating the results produced by the proposed methodology using the DRIVE and STARE databases, we have adopted the criterion initially established by Hoover [11] and afterward used by several other authors, which validates the estimated OD if positioned within 60 pixels of manually selected centers. For the INSPIRE-AVR images we have considered a disc radius of 


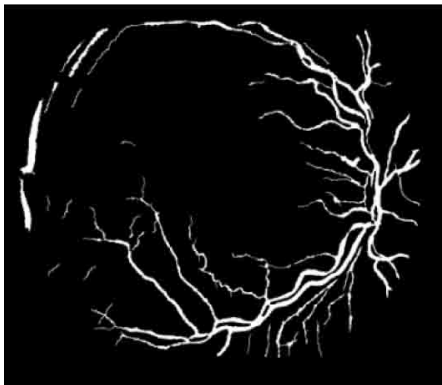

(a)

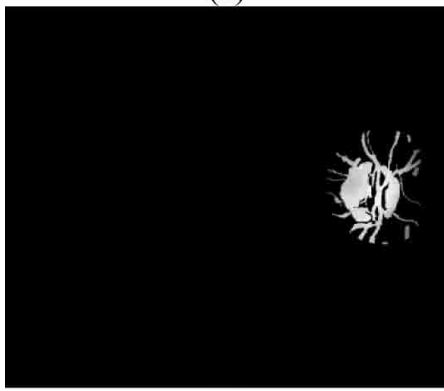

(d)

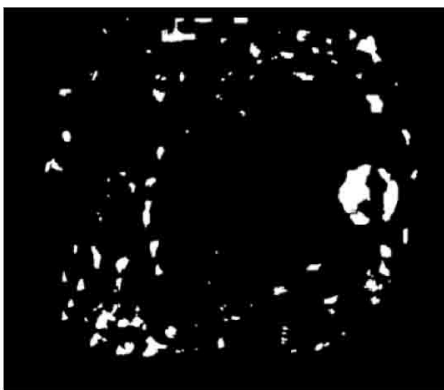

(b)

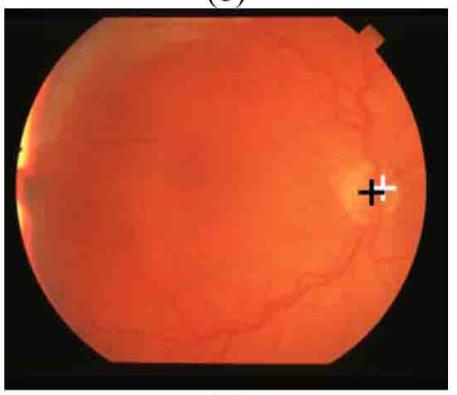

(e)

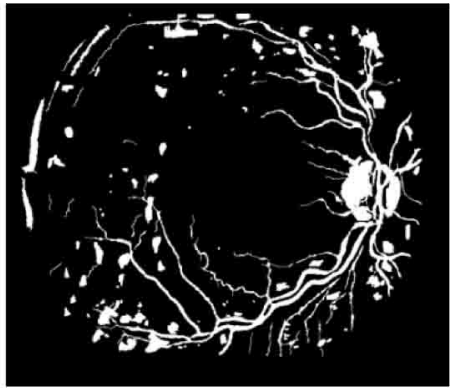

(c)

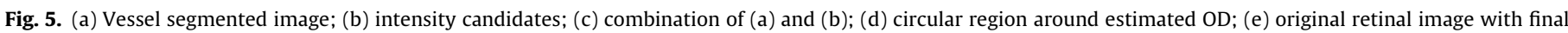
(black cross) and estimated (white cross) OD positions.

180 pixels as established in [25]. Due to the variability of the MESSIDOR images, the definition of one single criterion for disc diameter is not possible, so a detected position was considered valid if it was inside the OD; these results were assessed by the authors under the guidance of an expert.

The results obtained for the four databases are summarized in Table 1 . The numbers of successful OD detections with the proposed method are shown in the third column of this table. In order to clarify the influence on OD detection of the two main criteria (entropy and intensity) which are combined in the proposed methodology, we have applied each criterion separately, and we have considered as OD position the image location where the maximum value for the criterion is achieved. The two last columns of the table contain the number of successful detections where the OD location was associated with the position of the absolute maximum of entropy (fourth column) and with the absolute maximum of intensity (fifth column). From the analysis of the values in Table 1, we can conclude that both entropy and intensity are useful features for OD location; however, these features are sensitive to image quality and to the occurrence of pathological conditions, which have a significant influence on the performance of disc detection. Nevertheless, the combination of information from both features improves the robustness of OD localization, as clearly demonstrated by the results obtained using the proposed methodology.

Table 2 details the results of the combination of entropy and intensity, by indicating the number of images where the OD has been successfully located in each phase of the algorithm. Besides the total number of correctly detected OD positions, in each row are presented the number of images where the final OD location corresponds to the initial estimation, and the correct OD locations that were accomplished in the validation phase, either by the maximum

Table 1

Results of the proposed method compared with the stand-alone detection based on the maximum of entropy and the maximum of intensity.

\begin{tabular}{|c|c|c|c|c|}
\hline Database & $\begin{array}{l}\text { Number of } \\
\text { images }\end{array}$ & $\begin{array}{l}\text { Number (percentage) of } \\
\text { successful OD locations }\end{array}$ & $\begin{array}{l}\text { Absolute maximum of } \\
\text { entropy (reduced } \\
\text { resolution images) }\end{array}$ & $\begin{array}{l}\text { Absolute } \\
\text { maximum of } \\
\text { intensity }\end{array}$ \\
\hline DRIVE & 40 & $40(100 \%)$ & 39 & 37 \\
\hline STARE & 81 & $80(98.8 \%)$ & 63 & 58 \\
\hline MESSIDOR & 1200 & $1197(99.8 \%)$ & 1192 & 1155 \\
\hline INSPIRE-AVR & 40 & $40(100 \%)$ & 40 & 38 \\
\hline
\end{tabular}

Table 2

Criteria for OD localization.

\begin{tabular}{|c|c|c|c|c|}
\hline \multirow[t]{2}{*}{ Database } & \multirow{2}{*}{$\begin{array}{l}\text { Number of } \\
\text { successful OD } \\
\text { locations }\end{array}$} & \multirow{2}{*}{$\begin{array}{l}\text { Initial } \\
\text { estimation } \\
\text { Maximum of entropy in } \\
\text { intensity candidates (reduced } \\
\text { resolution images) }\end{array}$} & \multicolumn{2}{|l|}{ Validation } \\
\hline & & & $\begin{array}{l}\text { Maximum of entropy in } \\
\text { intensity candidates }\end{array}$ & $\begin{array}{l}\text { Maximum of intensity in } \\
\text { largest area segment }\end{array}$ \\
\hline DRIVE & 40 & 40 & 0 & 0 \\
\hline STARE & 80 & 74 & 1 & 5 \\
\hline MESSIDOR & 1197 & 1193 & 3 & 1 \\
\hline INSPIRE-AVR & 40 & 40 & 0 & 0 \\
\hline
\end{tabular}



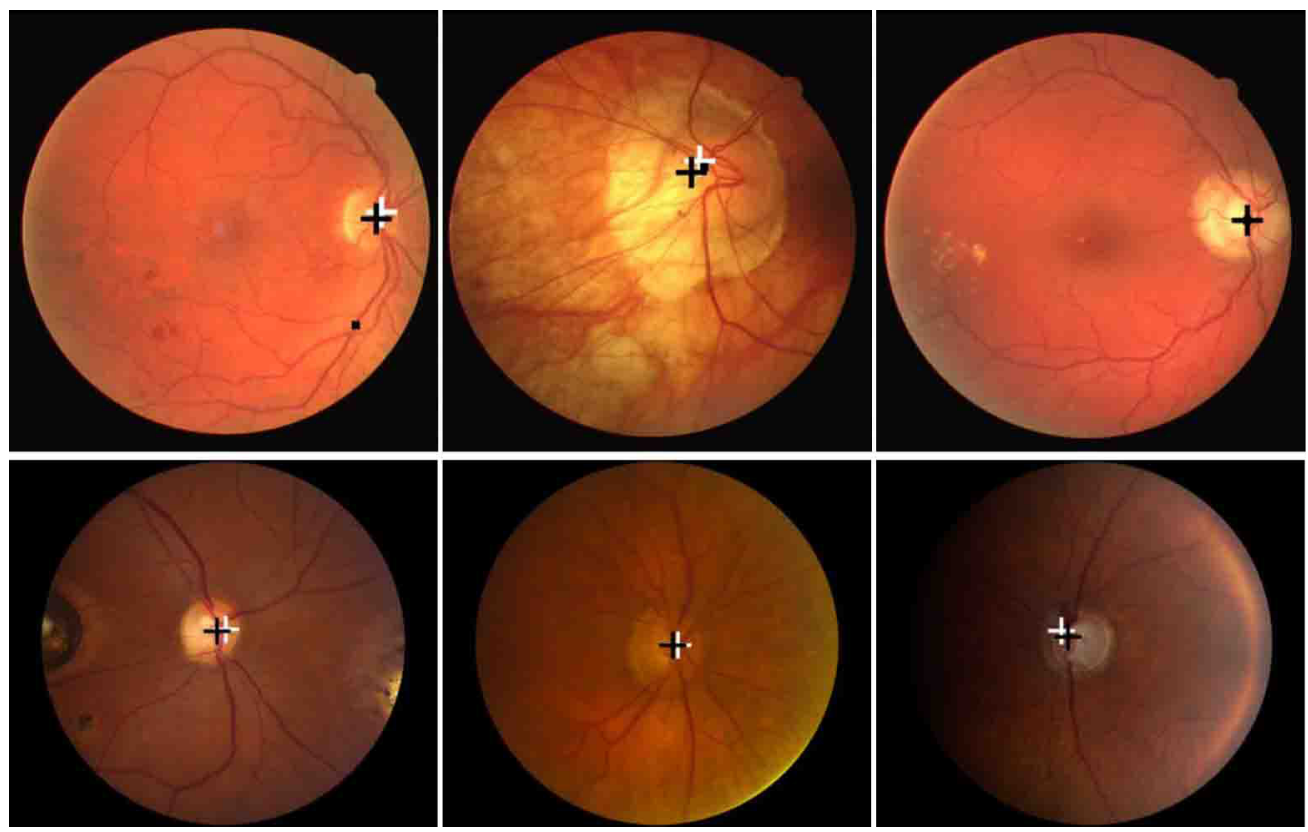

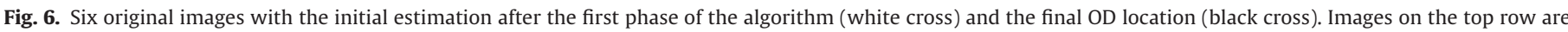
from DRIVE database while the bottom row shows images from the INSPIRE-AVR database.

value of entropy calculated on the original images (following the detection of several local maxima of entropy) or by the maximum value of intensity in the largest segment (when the entropy values were not reliable).

Table 3 compares the results of our method with the performance of other solutions reported in the literature for the same datasets.

Fig. 6 shows some results for the DRIVE (top row) and INSPIREAVR (bottom row) images. In the top row, we can observe on the left the single case where the absolute maximum of entropy was not associated with the OD location, while in the center is presented the single image where the intensity maximum did not belong to the OD; in both cases, the proposed method was able to achieve a correct solution. Finally, in the rightmost image the absolute maximum of entropy is coincident with the final OD location. The two leftmost images in the bottom row of Fig. 6 are the INSPIRE-AVR images where the highest intensity pixels do not coincide with the disc. In all these images the black dot marks the position of the absolute maximum of entropy, and the white and black crosses are the initial and final OD positions, respectively.

Fig. 7 presents the results of OD localization in six STARE images. The top-left image is the only image where the algorithm failed OD localization. Fig. 7(b) and (c) show two images where the initial estimation (first phase) got a wrong solution, but a correct position was achieved in the second phase where the final OD position is associated with to the centroid of the largest segment in the intensity

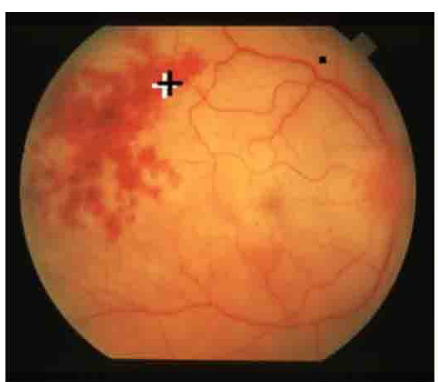

(a)

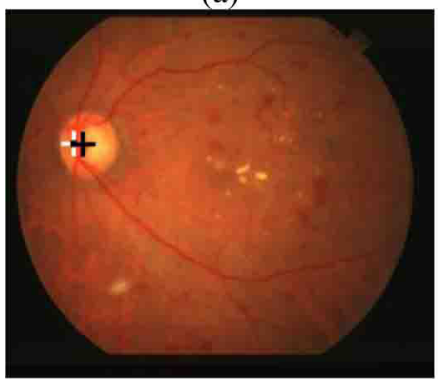

(d)

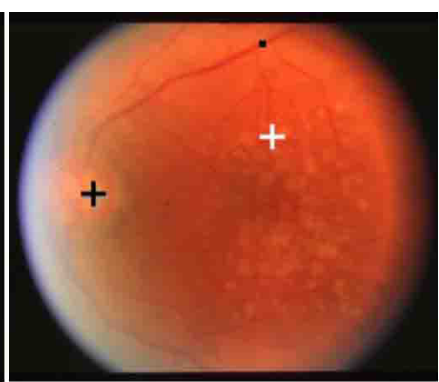

(b)

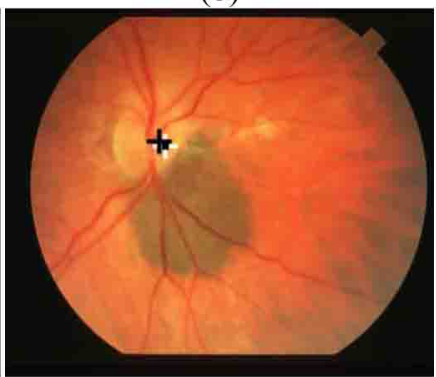

(e)

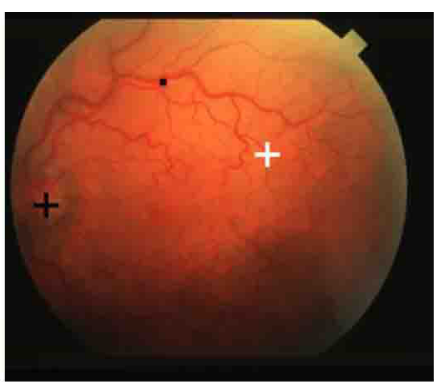

(c)

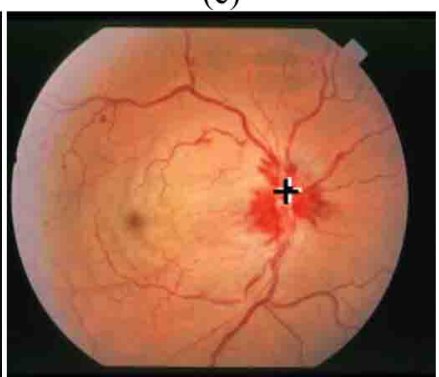

(f)

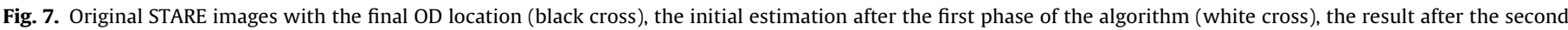

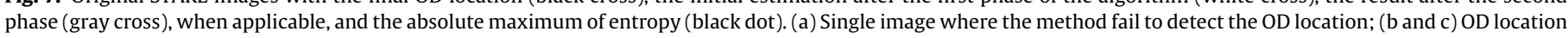
obtained in the second phase using the area and intensity criteria; ( $d-f)$ OD location calculated using low resolution images. 


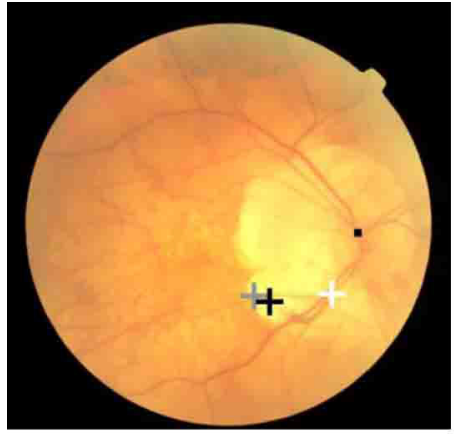

(a)

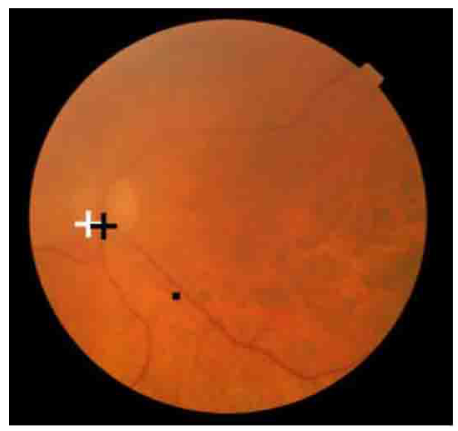

(d)

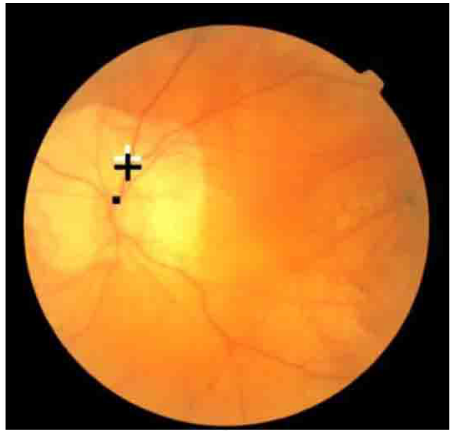

(b)

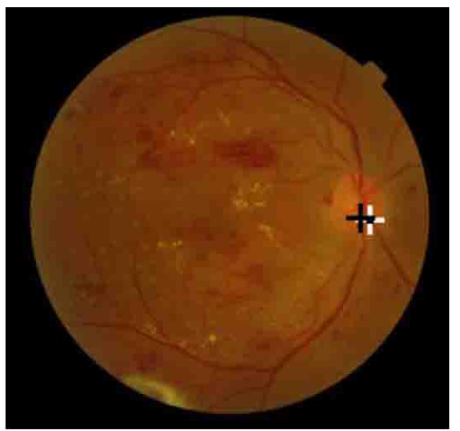

(e)

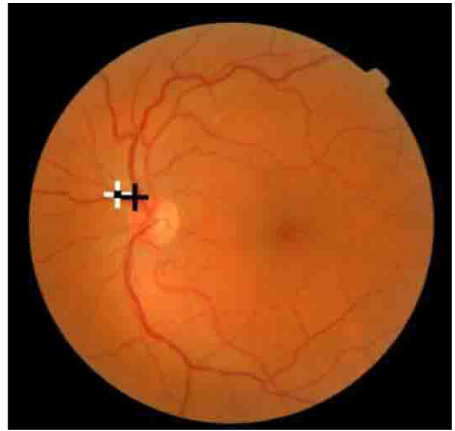

(c)

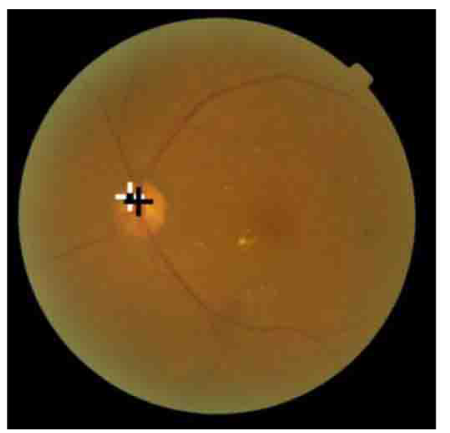

(f)

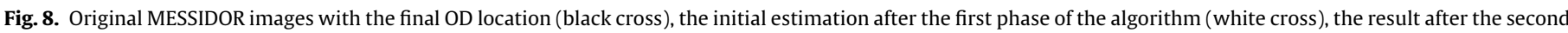

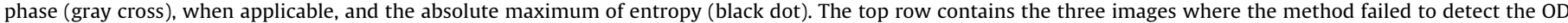
location, while the bottom row shows successful results.

Table 3

Performance of methods for OD localization.

\begin{tabular}{llc}
\hline Method & Database & $\begin{array}{l}\text { Percentage of } \\
\text { correct OD } \\
\text { locations }\end{array}$ \\
& & $100 \%$ \\
Proposed method & DRIVE & $98.8 \%$ \\
& STARE & $99.8 \%$ \\
Hoover [11] & MESSIDOR & $89.0 \%$ \\
Foracchia [12] & STARE & $97.5 \%$ \\
Haar [13] & STARE & $93.8 \%$ \\
Perez-Rovira [16] & STARE & $91.4 \%$ \\
Youssif [14] & STARE & $100 \%$ \\
& DRIVE & $98.8 \%$ \\
Lu[6] & STARE & $98.8 \%$ \\
& STARE & $99.8 \%$ \\
\hline
\end{tabular}

segmented image. A successful estimation of OD position using low resolution images was obtained for each one of the three images in the bottom row of Fig. 7 .

The three images of the MESSIDOR database where the proposed algorithm failed at localizing the OD are depicted in the top row of Fig. 8. For the images on the left and center, the absolute maximum of entropy is inside the disc, but the disc area was not included in the intensity image; the largest area candidate was selected in the leftmost image, while in the image shown in the center the initial OD position (white cross) was refined but could not reach the disc. In the third image, a final position near the border of the OD was finally selected.

\section{Conclusions}

A methodology for automating the localization of the OD in color images of the retina was described, based on the combination of vascular and intensity information. The new measure of entropy of vascular directions proved to be a good solution for assessing vessel convergence, which is one major feature of the optic disc region in a retinal image. Although for most normal and pathological images the simple detection of the absolute maximum of entropy is sufficient for correctly localizing the OD, the robustness of the method was increased by including additional intensity constraints.

The performance of the method herein described was greatly improved by the determination of an initial OD position using a low resolution entropy map, and further by the inclusion of a subsequent validation phase where an alternative solution is searched for if the calculated entropy values are not considered reliable. A significant reduction in computation time, and the attainment of a useful OD localization even in images where either the disc intensity contrast or the quality of the segmented vasculature is low, are the most relevant improvements. The inclusion of a refinement phase also allowed a final position that in general is nearer to the ideal OD center.

The method was evaluated using images from four databases with very promising results, outperforming most of the previouslyknown solutions. The OD location obtained with this automatic approach will be used as an initial step for the complete segmentation of the disc area.

\section{Acknowledgment}

We want to thank the authors of the four public databases for making their images available This work was supported by FEDER funds through the Programa Operacional Factores de Competitividade - COMPETE and by Portuguese funds through FCT - Fundação para a Ciência e a Tecnologia in the framework of the project PEstC/SAU/LA0002/2011.

\section{References}

[1] Jelinek H, Cree M. Automated image detection of retinal pathology. Boca Raton: CRC Press, Taylor and Francis Group; 2010. 
[2] Couper DJ, Klein R, Hubbard LD, Wong TY, Sorlie P, Cooper LS, et al. Reliability of retinal photography in the assessment of retinal microvascular characteristics: The atherosclerosis risk in communities study. Am J Ophthalmol 2002;133(1):78-88.

[3] Sinthanayothin C, Boyce JF, Cook HL, Williamson TH. Automated localization of the optic disc, fovea, and retinal blood vessels from digital colour fundus images. Br J Ophthalmol 1999;83:902-10.

[4] Aquino A, Gegúndez-Arias ME, Marin D. Detecting the optic disc boundary in digital fundus images using morphological, edge detection and feature extraction techniques. IEEE Trans Med Imaging Nov 2010;29(11):1860-9.

[5] Aquino A, Gegúndez-Arias ME, Marin D. Automated optic disc detection in retinal images of patients with diabetic retinopathy and risk of macular edema. Int J Biol Life Sci 2012;8(2):87-92.

[6] Lu S. Accurate and efficient optic disc detection and segmentation by a circular transform. IEEE Trans Med Imaging 2011;30(12):2126-33.

[7] Lalonde M, Beaulieu M, Gagnon L. Fast and robust optic disc detection using pyramidal decomposition and Hausdorff-based template matching. IEEE Trans Med Imaging Nov 2001;20(11):1193-200.

[8] Osareh A, Mirmehdi M, Thomas B, Mrkham R. Classification and localisation of diabetic-related eye disease. In: Heyden A, et al., editors. ECCV 2002. Heidelberg: Springer; 2002. p. 502-16.

[9] Lowell J, Hunter A, Steel D, Basu A, Ryder R, Fletcher E, et al. Optic nerve head segmentation. IEEE Trans Med Imaging 2004;23(February (2)):256-64.

[10] Abdel-Ghafar RA, Morris T. Progress towards automated detection and characterization of the optic disc in glaucoma and diabetic retinopathy. Med Inform Internet Med 2007;32(1):19-25.

[11] Hoover A, Glodbaum M. Locating the optic nerve in a retinal image using the fuzzy convergence of the blood vessels. IEEE Trans Med Imaging 2003;22(August (8)):951-8.

[12] Foracchia M, Grisan E, Ruggeri A. Detection of optic disc in retinal images by means of a geometrical model of vessel structure. IEEE Trans Med Imaging 2004;23(October (10)):1189-95.

[13] Haar F. Automatic localization of the optic disc in digital colour images of the human retina. University of Utrecht; 2005 [M.Sc thesis].

[14] Youssif A, Ghalwash AZ, Ghoneim A. Optic disc detection from normalized digital fundus images by means of a vessels' direction matched filter. IEEE Trans Med Imaging 2008;27(January (1)):11-8.

[15] Niemeijer M, Abràmoff MD, van Ginneken B. Fast detection of the optic disc and fovea in color fundus photographs. Med Image Anal 2009;13:859-70.

[16] Perez-Rovira A, Trucco E. Robust optic disc location via combination of weak detectors. In: 30th Annual International IEEE EMBS Conference. 2008. p. 3542-5.

[17] Xu X, Gravin MK, Abràmoff MD, Reinhardt JM. Simultaneous automatic detection of the optic disc and fovea on fundus photographs. In: Dawant BM, Haynor DR, editors. Proc. SPIE medical imaging 2011: image processing, vol. 7962. 2011. p. $7962 \mathrm{~T}$.

[18] Mendonça A, Cardoso F, Sousa A, Campilho A. Automatic localization of the optic disc in retinal images based on the entropy of vascular directions. In: Image analysis and recognition; 2012. p. 424-31 [LNCS 7325].

[19] Image Science Inst., http://www.isi.uu.nl/Research/Databases/DRIVE
[20] The STARE project, http://www.ces.clemson.edu/ ahoover/stare

[21] MESSIDOR project, http://messidor.crihan.fr/

[22] Niemeijer M, Xu X, Dumitrescu A, Gupta P, van Ginneken B, Folk J, et al. Automated measurement of the arteriolar-to-venular width ratio in digital color fundus photographs. IEEE Trans Med Imaging 2011;30(November (11)).

[23] Mendonça AM, Campilho AC. Segmentation of retinal blood vessels by combining the detection of centerlines and morphological reconstruction. IEEE Trans Med Imaging 2006;25(September (9)):1200-13.

[24] Chaudhuri S, Chattererjee S, Katz N, Glodbaum M. Detection of blood vessels in retinal images using two-dimensional matched filters. IEEE Trans Med Imaging 1989;8(March (3)):263-9 [datasets II, ICML, Washington].

[25] Niemeijer M, Xu X, Dumitrescu A, Gupta P, van Ginneken B, Folk J, et al. Automated measurement of the arteriolar-to-venular width ratio in digital color fundus photographs. IEEE Trans Med Imaging 2011;30(11): $1941-50$.

Ana Maria Mendonça received the Ph.D. degree in Electrical Engineering from the Faculty of Engineering, University of Porto (FEUP) in 1994. Currently, she is Associate Professor at the Department of Electrical and Computer Engineering at the Faculty of Engineering, University of Porto, Portugal. She joined INEB - Institute for Biomedical Engineering - in 1989, where she has been a researcher of the Bioimaging Group. Her research interests include image processing and analysis, and, more specifically, medical image analysis.

António V.Sousa received the Ph.D. degree in Engineering Sciences from the Faculty of Engineering, University of Porto (FEUP) in 2008. Presently, he is Adjoined Professor at the Department of Mathematics of ISEP, Polytechnic Institute of Porto, Portugal. He is a researcher of the Bioimaging Group of INEB. His research interests include image processing and classification.

Luís Mendonça is a board certified ophthalmologist from the Hospital de Braga (Braga, Portugal). He received his MD degree (1999-2005) from the Faculty of Medicine of the University of Porto and completed his ophthalmology residency (2007-2010) at the Department of Ophthalmology of Hospital São João (Porto, Portugal). He attended a Retinal Research Fellowship (2010-2011) at LuEsther T. Mertz Retinal Research Centre/Vitreous Retina Macula Consultants of New York (New York, NY, USA) and has clinical and research interest in retinal and choroidal disorders such as diabetic retinopathy, age-related macular degeneration and retinal vascular occlusions.

Aurélio Campilho received the Ph.D. degree in Electrical Engineering in 1985. Currently, he is a Professor in the Department of Electrical and Computer Engineering at the Faculty of Engineering, University of Porto, Portugal. From 1994-2000 he served as chairman of INEB - Biomedical Engineering Institute. For several years, he also served as president of APRP - Portuguese Association for Pattern Recognition (member of IAPR) and President of the General Assembly of APRP. He is the coordinator of the Bioimaging research group of INEB. His research interests are in the area of medical image analysis, image processing and computer vision. He organized several special issues and conferences. He served as Associate Editor of IEEE TBME and MVA journals. He is chairman of the series of conferences ICIAR. 